\title{
THE SATELLITES OF URANUS
}

by

Dale P. Cruikshank

\begin{abstract}
Institute for Astronomy, University of Hawaii
2680 Woodlawn Drive, Honolulu, Hawaii 96822
\end{abstract}

\section{ABSTRACT}

The Uranian satellite system contains five known members, all of which are difficult to study owing to their faintness and proximity to Uranus. The photometry of these objects is not in a satisfactory state, nor is the photovisual spectrophotometry. Infrared observations are in some sense easier and more precise because Uranus itself is faint in the near Infrared and interferes but little with the satellite studies. The near infrared work reveals water ice or frost on the satellite surfaces, perhaps in a very pure state. The satellites are most similar to Ganymede in terms of the strength of the ice bands, but subtle differences, now under study, may be present. The diameters and masses of the satellites, while not readily measurable directly, can be estimated from generalizations about the surface geometric albedos, assumed mean densities, and certain dynamical arguments. Most of the data and theories are consistent with bodies with radii in the range $160-520 \mathrm{~km}$ (similar to the larger asteroids) and albedos on the order of 0.5 , consistent with ice and snow. The mean densities are probably similar to those of the icy Saturn satellites, about 1.3 $\mathrm{g} / \mathrm{cm}^{3}$. Uranus' satellites probably formed after the event that caused the planet to tilt to the presently observed obliquity.

\section{INTRODUCTION}

Following William Herschel's discovery of the planet Uranus in 1781, he continued to build larger and better telescopes, culminating in the great 40-foot telescope at Slough. With a telescope of 18.7 inches aperture he found the outer two satellites, U3 Titania, and $\mathrm{U} 4$ Oberon, in 1787, and then searched for additional satellites 
for the next 14 years. Though he eventually announced the discovery of four additional satellites (in 1798), subsequent observations by others suggested that the positions and periods of revolution given by Herschel were spurious. William Lasse1l, the discoverer of Triton, observed the planet with telescopes of probably higher quality (better rejection of scattered light from the planet), and reported in 1851 the discovery of U2 Umbriel and U1 Ariel. In a detailed examination of the circumstances of discovery, $D$. Rawlins in a paper at this conference has given evidence that Herschel should be given credit for the discovery of Umbriel in 1801 and Otto Struve credit for Ariel in 1847, though the essential work of Lassell in firmly establishing the orbital periods is not doubted. Additional historical analysis of the question of satellite discoveries will be found in Alexander (1965). Sir John Herschel suggested the names that are now in use for the first four sateliites (see Barton, 1946). Oberon and Titania are the king and queen of the fairies in Shakespeare's Midsummer Night's Dream.

Ariel and Umbriel appear in Pope's Rape of the Lock, and Ariel is "an airy, tricksy spirit" in Shakespeare's Tempest.

The last satellite discovery for Uranus was that of Miranda by G. P. Kuiper in February, 1948. He found the innermost satellite at magnitude 16.5 on a four-minute photographic exposure with the 82-inch McDonald Observatory telescope, at that time the largest telescope with which a detailed survey of satellite systems had been conducted. The same survey revealed N2 Nereid in 1949. Kuiper (1949) named U5 Miranda after a cherub in the Tempest. The orbital data and visual magnitudes of the five known satellites are given in Table $I$. The orbital data are taken from Morrison and Cruikshank (1974) who extracted them from Gondolatsch (1965) and Whitaker and Greenberg (1973). 
TABLE I. BRIGHTNESS AND ORBITAL PROPERTIES OF THE URANIAN SATELLITES

\begin{tabular}{|c|c|c|c|c|c|c|}
\hline & $\begin{array}{l}\text { Visual } \\
\text { Brightness } \\
\text { (mean } \\
\text { opposition) }\end{array}$ & {$\left[10^{3} \mathrm{~km}\right]$} & $\begin{array}{l}\text { Radius } \\
\text { (plane- } \\
\text { tary } \\
\text { radii) }\end{array}$ & $\begin{array}{l}\text { Perfod } \\
\text { (days) }\end{array}$ & $\begin{array}{l}\text { Eccen- } \\
\text { tricity }\end{array}$ & $\begin{array}{l}\text { Incli- } \\
\text { nation } \\
\text { (degrees) } \\
\end{array}$ \\
\hline U5 Miranda & 16.5 & 130 & 5.13 & 1.4135 & 0.017 & 3.4 \\
\hline U1 Ariel & 14.4 & 192 & 7.54 & 2.520 & 0.0028 & 0 \\
\hline U2 Umbrie1 & 15.3 & 267 & 10.5 & 4.144 & 0.0035 & 0 \\
\hline U3 Titania & 14.0 & 438 & 17.2 & 8.706 & 0.0024 & 0 \\
\hline U4 Oberon & 14.2 & 586 & 23.0 & 13.46 & 0.0007 & 0 \\
\hline
\end{tabular}

All five satellites and Uranus are nicely shown in the composite photograph in Figure 1 provided by William Sinton (1972).
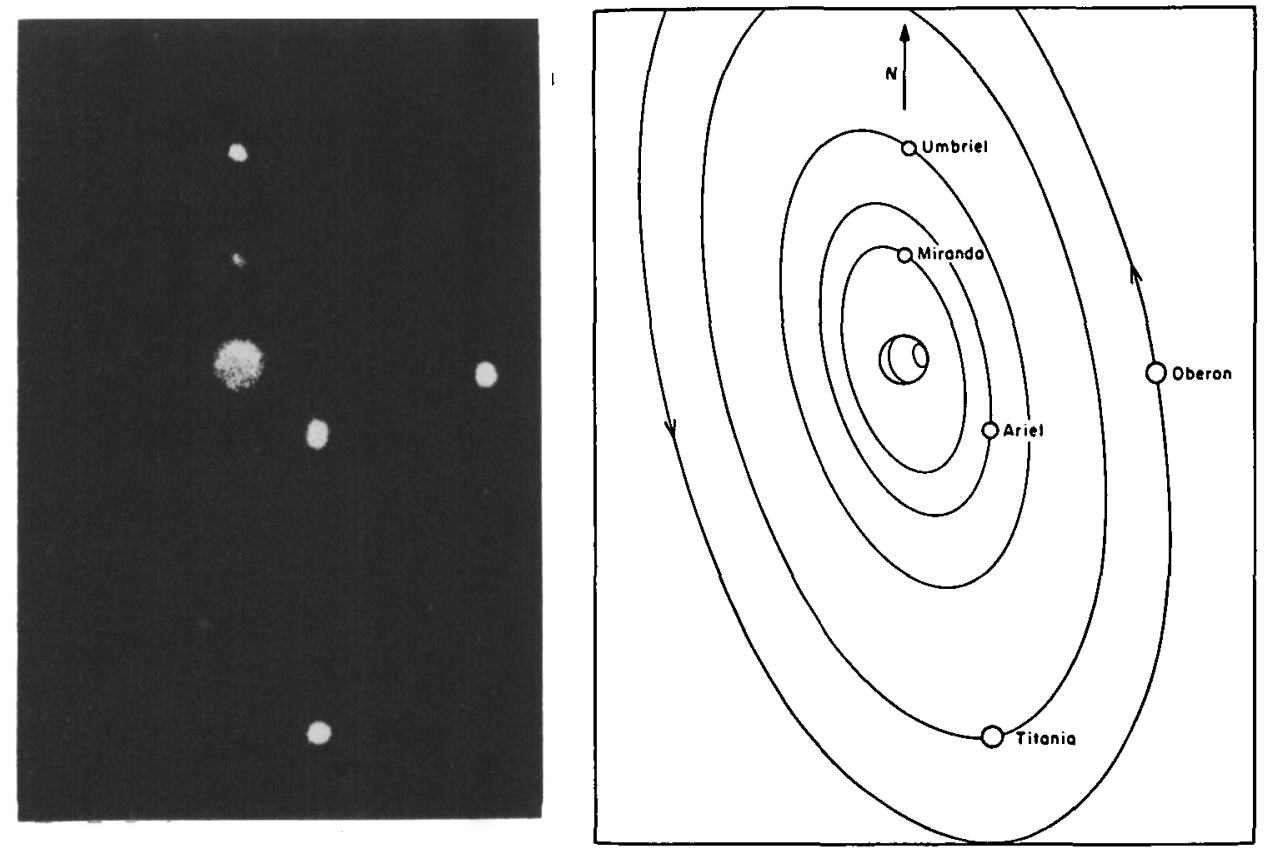

Figure 1. The composite photograph on the left shows Uranus and the five known satellites, as identified in the guide on the right. The Uranus image is a composite of six individual oneminute exposures on 17 March and 7 May, 1972, while the satellites were recorded on an exposure of about one hour on 17 March. The exposures of the planet were made through a filter transmitting in the region of a strong methane band which is heavily absorbed in the Uranus spectrum. The nonuniform density of the Uranus image is real and is related to the distribution of haze in the upper atmosphere of the planet. From Sinton (1972). 


\section{Photometry}

The earliest photometric work on the Uranian satellites was the set of broadband measurements on Titania and Oberon by Harris (1961) with the 82-inch McDonald reflector. The circumstances of the observations were never published, but have to some degree been reconstructed by Andersson (1974). New measurements of the same two satellites by Andersson, Degewij and Zellner have just appeared (Degewij, et al. 1980a); their values of $V(1,0)$ are given in Table II.

TABLE II. PHOTOMETRY OF THE URANIAN SATELLITES

\begin{tabular}{lccc}
\hline Satellite & $\begin{array}{c}\text { Harris } \\
(1961)\end{array}$ & $\begin{array}{c}\text { Reitsema et al. } \\
(1978)\end{array}$ & $\begin{array}{c}\text { Degewij et al. } \\
(1980 \mathrm{a}, \mathrm{b})\end{array}$ \\
\hline U5 Miranda & $3.8^{\mathrm{a}}$ & $3.79 \pm .17$ & - \\
U1 Ariel & $1.7^{\mathrm{a}}$ & $1.48 \pm .15$ & - \\
U2 Umbriel & $2.6^{\mathrm{a}}$ & $2.31 \pm .15$ & - \\
U3 Titania & $1.3^{\mathrm{b}}$ & $1.27 \pm .14$ & $1.27 \pm .14^{\mathrm{b}}$ \\
U4 Oberon & $1.5^{\mathrm{b}}$ & $1.42 \pm .15$ & $1.52 \pm .05^{\mathrm{b}}$ \\
\hline
\end{tabular}

\section{Notes:}

${ }^{\text {a }}$ Photographic estimate.

$b_{\text {Photoelectric measurement. }}$

${ }^{c}$ Photometry from Charge-Coupled Device (CCD) images.

The magnitudes of the other three satellites in Harris' listing are photographic estimates based on plates obtained largely by $\mathrm{T}$.

Gehrels; direct photometric measurements, even with large telescopes, are very difficult because of the proximity of Uranus. Additional differential photometry of all five satellites was obtained by Reitsema et al. (1978) using a charge-coupled device (CDD) yielding images of the satellite system through four different color filters, including the broadband $V$ filter. The Reitsema et al. (1978) data were referenced to the brightest object, Titania. In Table II, I have used the Degewij et $a 1 .(1980 a, b)$ value of $v(1,0)$ for Titania with its standard error of \pm 0.14 , and list the resulting Reitsema et al. (1978) values from their published differential values, propagating their estimated errors. Shown also are the earlier values 
from Harris (1961), though no error estimates are available for these data. It can be seen that the Degewij et al. $(1980 \mathrm{a}, \mathrm{b})$ and Reitsema et al. (1978) values for Oberon do not agree as well as one might hope for objects as bright as these and at relatively large distances from the planet (Titania is about 34 arcsec from Uranus and Oberon is about $45 \mathrm{arcsec}$ ). They do agree within the values of the error bars, however. The agreement with the Harris photometry is good for Titania, Oberon, and Miranda, though Ariel and Umbriel are not in good accord. My colleagues who are familiar with CCD imagery tell me that photometry with these systems has not reached its full degree of perfection, which may account for part of the difficulty in Table II, but the differences in the Harris values and the more recent data may in part result from the different viewing geometry at the epochs of the two sets of observations, the more recent representing a more nearly polar view of the satellite system. Note that the photometry summary given by Cruikshank (1980, Table II) used values of Titania and Oberon from Andersson's (1974) thesis. His data have been superceded by the new analysis in Degewij et al. (1980a), though the differences in $v_{o}[$ or $\mathrm{V}(1,0)]$ are small. Table III gives a summary of the meager information at hand.

TABLE III. PHOTOMETRIC COLORS OF THE URANIAN SATELLITES

\begin{tabular}{lll}
\hline & $\mathrm{U}-\mathrm{B}$ & $\mathrm{B}-\mathrm{V}$ \\
\hline Harris & & \\
Titania & 0.25 & 0.62 \\
Oberon & 0.24 & 0.65 \\
Degewij et $a l$. & & \\
Titania & 0.28 & 0.68 \\
Oberon & 0.22 & 0.69 \\
Reitsema et al. & \\
"Umbriel, Titania, and Miranda are very similar \\
(0.5 - 1.0 $\mu \mathrm{m})$, while Ariel and Oberon form a \\
second spectrai reflectance group which is \\
slightly less red."
\end{tabular}

Broadband photometry in the $\mathrm{J}(1.25 \mu \mathrm{m}), \mathrm{H}(1.60 \mu \mathrm{m})$, and $\mathrm{K}$ $(2.2 \mu \mathrm{m})$ bands can, in principle, help distinguish solar system 
bodies of icy or rocky surface composition (Morrison et al. 1976; Cruikshank 1980; Degewij et al. 1980b; Hartmann et al. 1981 and others). The J-H and $\mathrm{H}-\mathrm{K}$ colors for icy objects tend to be distinctly different from those of rocky bodies, such as asteroids and low-albedo planetary satellites, but the colors of the Uranian satellites fall in a portion of the $\mathrm{J}-\mathrm{H}, \mathrm{H}-\mathrm{K}$ color diagram that is a bit ambiguous and characterized by nearly neutral coloration. This information, combined with the relatively neutral colors in the photovisual spectral region, leaves a serious ambiguity in the deduction of surface composition, though this has been clearly resolved by spectrophotometry in the near infrared, as discussed below. The broadband JHK photometry of the Uranian satellites has been obtained by Cruikshank et al. (1977), Cruikshank (1980), and Nicholson and Jones (1980). The spectrophotometry is somewhat more interesting than the broadband photometry, and the photometry will therefore not be discussed here in any detail.

\section{Photovisual Spectrophotometry}

In this context the term photovisual refers to data obtained with electronic detectors in the spectral region $0.3-1.1 \mu \mathrm{m}$. The first such data with significant spectral resolution obtained for the Uranian satellites are those of Johnson et al. (1978) who used the Oke scanner on the Hale 5-m telescope. They found that the reflectances of Titania, Ariel, and Oberon drop significantly shortward of $0.5 \mu \mathrm{m}$, with the reflectance at longer wavelengths essentially flat for Ariel and Titania, while that of Oberon continued to rise significantly. In contrast, the reflectance of Umbriel was found to rise toward the violet. They also published data for Triton which are at variance with results of similar quality obtained by other means (Cruikshank et al 1979; Bell et al. 1979). The Johnson et a1. (1978) data for Titania and Oberon show a much steeper ultraviolet drop than is supported by the UBV photometry given in Table III. Further, multi-filter spectrophotometry by Bell et al. (1979) of Titania and Oberon is distinctly different from the Johnson et al. (1978) data in the sense that no 
strong violet absorption is present in the former results. It can easily be argued that systematic errors in observation or reduction most of ten result in the loss of light in some spectral region rather than the addition of light, and that such errors would produce an apparent absorption in a spectrum rather than a tendency toward spectral neutrality. The Bell et al. (1979) data for Titania and Oberon, shown in Figure 2 clearly reveal a rather neutral color throughout the spectral range covered.

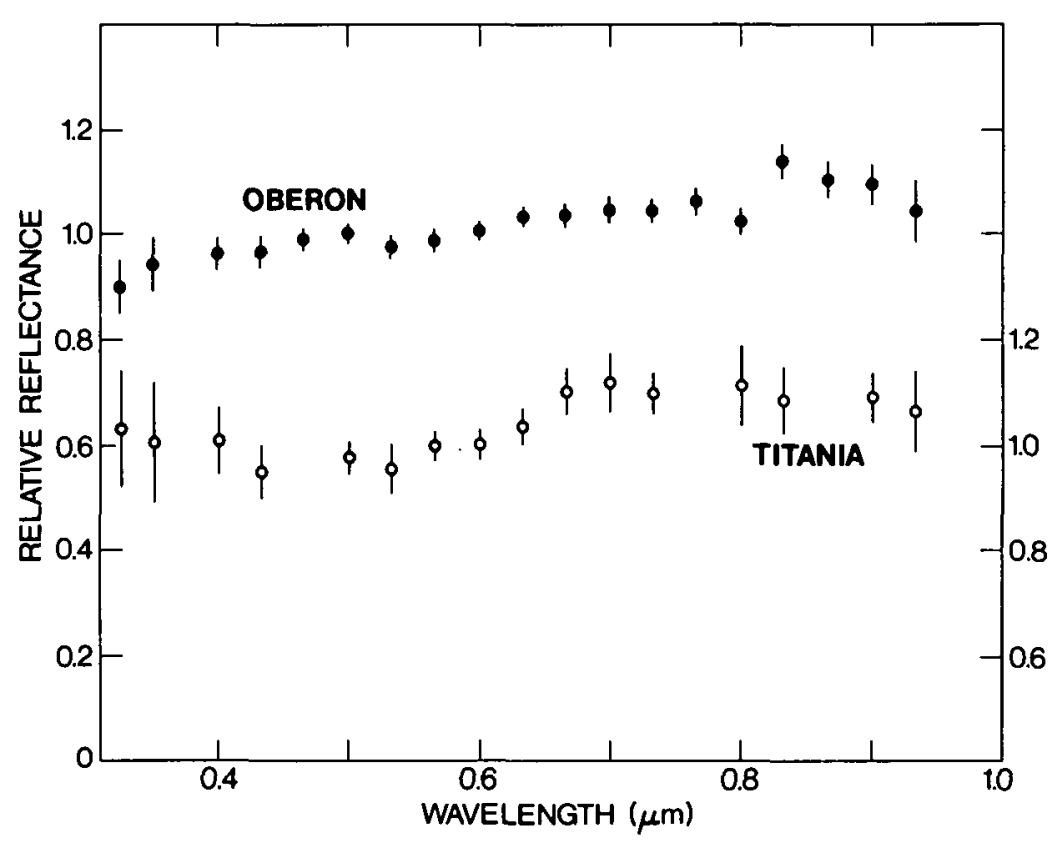

Figure 2. Reflectance spectra of Titania and Oberon, each normalized to unity at $0.56 \mu \mathrm{m}$. The reflectance scale on the left is that for Oberon and that on the right for Titania. From the work of Be11, et al. (1979), previously unpublished.

The disagreement between these two data sets, the strong coloration in the Johnson et al. (1978) data, and the latter's disagreement with the U-B, B-V colors, suggests that the Johnson et al. (1978) data are flawed by systematic effects. They were taken with a rather small aperture of 5 arcsec at the relatively large airmass (at least 1.36) forced by the southerly declination of Uranus. I 
suggest that differential atmospheric refraction caused the loss of the blue and violet light in their observations, resulting in what appears to be a strong photometric signature, and that their spectrophotometric curves are therefore spurious. For the present discussion, the relatively neutral reflectance of Titania and Oberon is regarded as fairly well established, while no satisfactory data on the other satellites as yet have been obtained.

\section{Near-Infrared Spectrophotometry}

I published the first infrared spectrophotometry of two Uranian satellites, Titania and Oberon, (Cruikshank 1980) and showed that their reflectances between 1.40 and $2.50 \mu \mathrm{m}$ reveal the strong absorptions (at $\sim 1.5,2.0$, and $2.4 \mu \mathrm{m}$ ) characteristic of water frost or ice. In the case of both satellites, the strengths of the absorption at $\sim 2.0$ and $2.4 \mu \mathrm{m}$ are less than in the spectra of the rings of Saturn and are quite comparable to those in the spectrum of Jupiter's Ganymede, as studied in great detail by Clark (1980) and Clark and McCord $(1980 \mathrm{a}, \mathrm{b})$. Apart from the $\mathrm{H}_{2} \mathrm{O}$ found in comets, the new measurements represent the greatest distance from the sun that water has been found in the solar system. Spectrophotometry, though crude, for Ariel and Umbriel was reported by Cruikshank and Brown (1981), in which it was shown that these satellites also have water frost or ice on their surfaces. R. H. Brown is studying the spectra of the Uranian satellites in detail as part of a Ph.D. thesis at the University of Hawaii. In the course of this work, he and I have obtained, in 1981, data of superior quality to those published in the two papers referenced. The significant improvement in the data has been achieved by the use of improved detectors and a much more satisfactory circular-variable interference filter (CVF) which provides the wavelength separation in the InSb photometric system used. The CVF used in the early work had a bandpass of $\sim 1 \%$, while the new filter has $5 \%$ and overall higher transmission.

To first order, the new 1981 data confirm the conclusions reached from the earlier studies, that is absorptions of $\mathrm{H}_{2} \mathrm{O}$ ice or frost dominate the near infrared spectrum with very strong absorptions at the appropriate wavelengths. In my 1981 paper with Mr. 
Brown we suggested that the spectrum of Unbriel shows ice bands of less strength than those of the other three satellites: While some differences among the new spectra are evident, it is not apparent that the earlier conclusion about Umbriel is borne out. The new data will permit a detailed comparison with laboratory spectra of ices and mixtures of ices and mineral contaminants, much in the way that Clark (1980) and Clark and McCord $(1980 \mathrm{a}, \mathrm{b})$ have done for the Galilean satellites and the rings of Saturn, and this will be substantially the direction of Mr. Brown's effort in the next year. A preliminary conclusion from the new data is that the spectra of the satellites do indeed differ from one another in subtle ways not discernable in the rougher data heretofore available, but clear trends or causes of the differences are not yet understood. The Interested reader is advised to watch for Mr. Brown's future publications on this subject.

In the meantime, I present here the published data in the form of Figures 3 and 4 . Figure 3 shows the spectrum of Titania (points with error bars) in two representations. At the top of the figure the solfd line is the spectrum of Saturn's rings and at the bottom it is the spectrum of Ganymede, in both cases provided by Dr. R. Clark. The Titania points are properly normalized to the respective comparison spectra by averaging over several points near $2.2 \mu \mathrm{m}$. This figure affirms the earlier statement that the Titania profile more closely resembles that of Ganymede than the rings of Saturn in terms of the depths of the distinct ice absorptions at $\sim 2$ and $2.4 \mu \mathrm{m}$. The new 1981 data noted above were obtained at Mauna Kea Observatory where the smaller airmass and drier sky permitted precise photometry right through the 1.9- $\mathrm{m}$ telluric water band, so the new data are not interrupted mid-spectrum as are the old, and the profile of the ice band at $\sim 2 \mu \mathrm{m}$ is we11 shown.

Published spectra for all four bright satellites of Uranus are given in Figure 4 with the Ganymede spectrum given for comparison in a11 cases. The spectra are arranged top to bottom in order of increasing apparent depth of the ice bands, though the new 1981 data may disrupt this ordering when they are fully analyzed. 


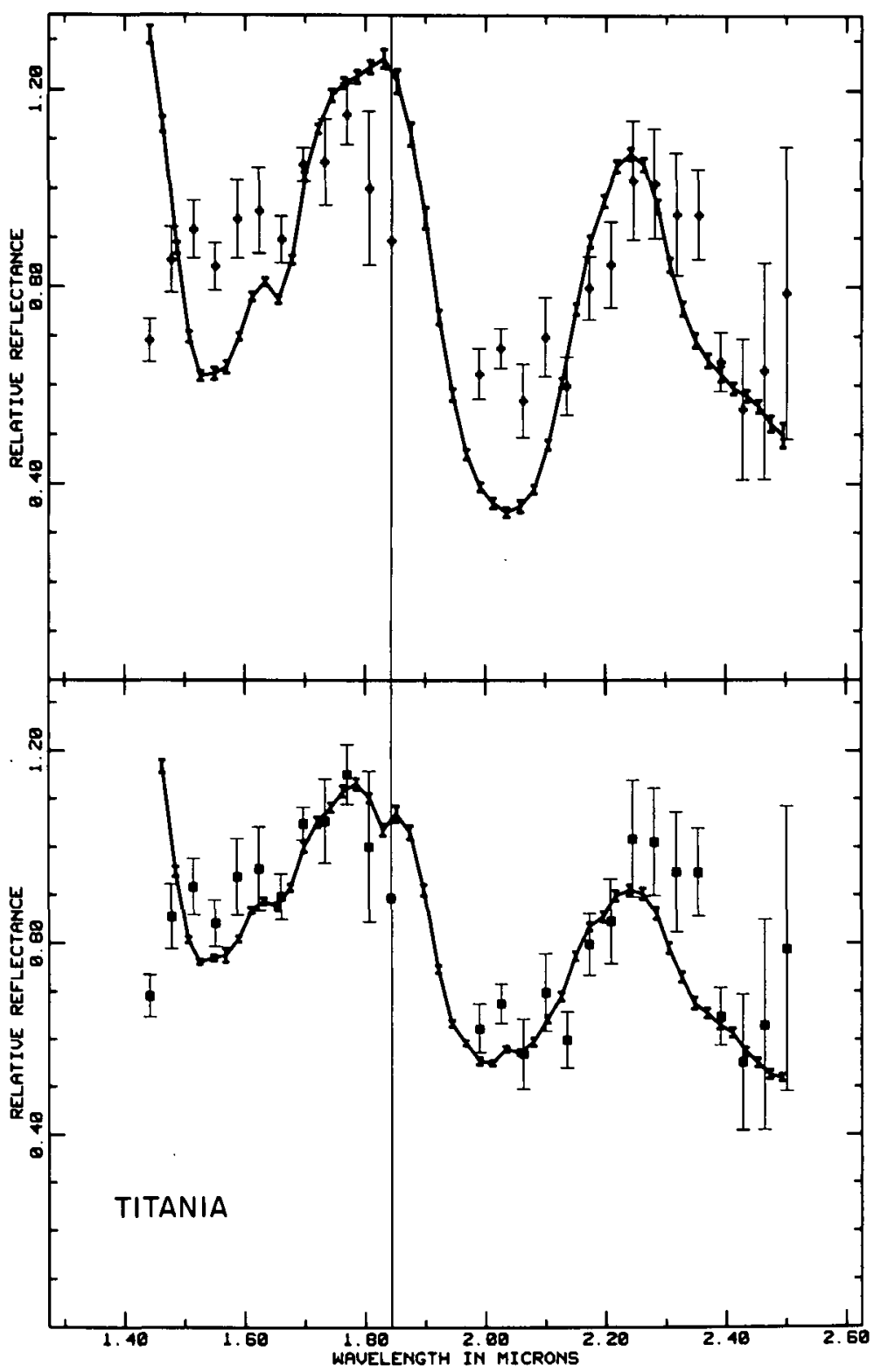

Figure 3. Spectrum of Titania (points with one-sigma error bars) compared with the spectrum of Saturn's rings (top) and the spectrum of Ganymede (bottom). Strong absorptions in the spectra of the rings, Ganymede, and Titania, are due to $\mathrm{H}_{2} \mathrm{O}$ ice or frost. From Cruikshank (1980). 


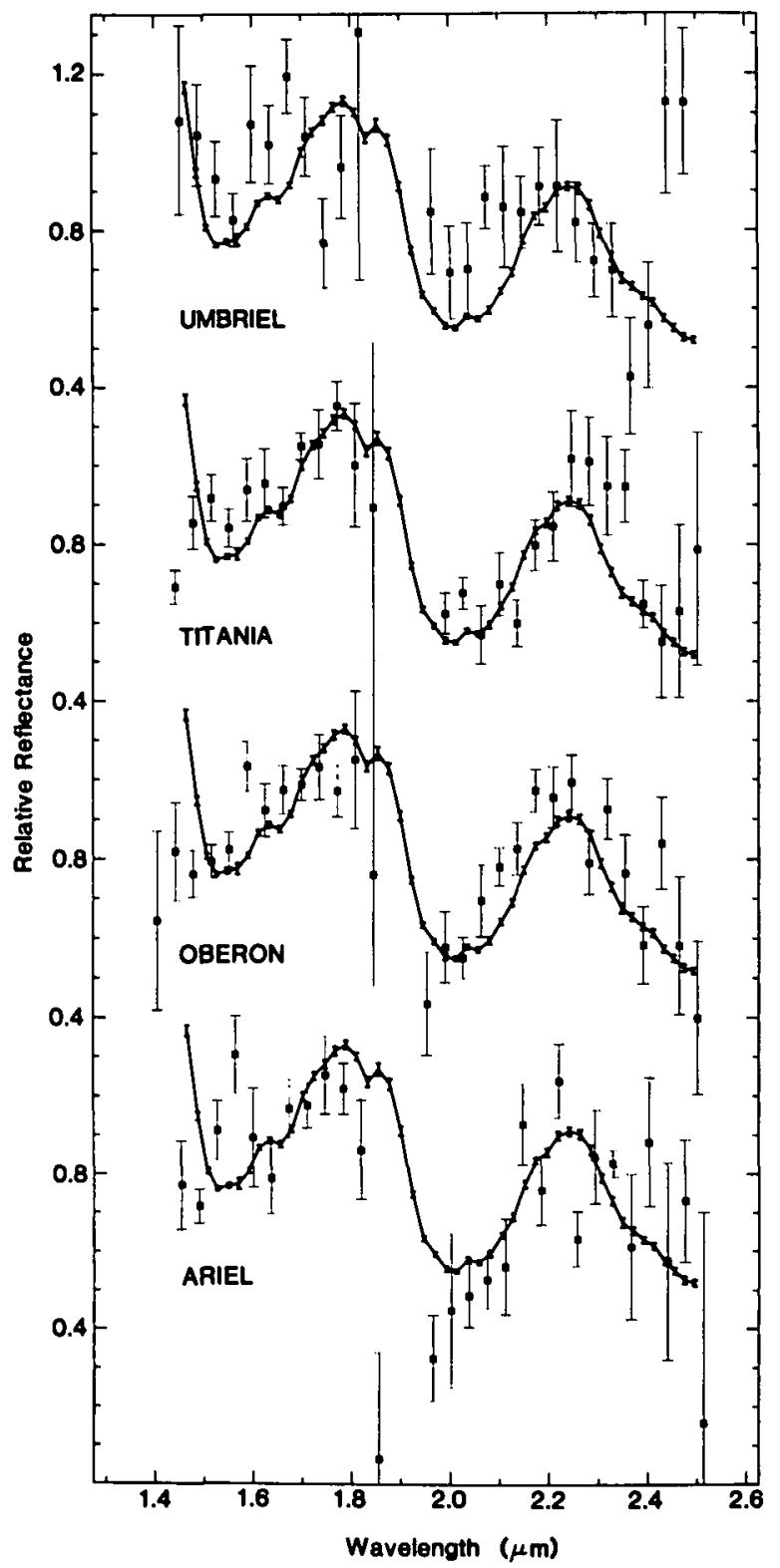

Figure 4. Spectra of the four outer Uranian satellites (points with one-sigma error bars) compared with the spectrum of Ganymede (solid lines). From Cruikshank and Brown (1981). 
The appearance of water frost in a relatively pure state (freedom from mineral contaminants) would support the apparent neutrality of the photovisual and near infrared spectra noted earlier from the broadband and photovisual spectrophotometry. Still, the ice bands are less deep than on Saturn's rings (regarded as quite pure by Clark and McCord, 1980b), and we have seen in Voyager pictures of Ganymede that its surface is quite varied and has evidence of geological structures suggesting materials in addition to ice on its surface. Further, the fact that the ice bands are weaker on Ganymede than on the rings suggest a contaminant filling in the bands. Clark (1980) concludes from an extensive laboratory study of ices and their contaminants that such mineral contaminants as occur. in the frost in Saturn's rings are not on the surfaces of the particles but a few micrometers to millimeters under the uppermost surface where they can lower the continuum level a small amount but not strongly affect the band depths. In contrast, mineral grains on Ganymede occur at the surface of the fine-grained frost layer, where they affect both the intensity of the continuum and the strength of the absorption bands. On the other hand, Ganymede shows a strong violet absorption in its spectrum, probably as a consequence of these mineral contaminants, and we have argued above that the Uranian satellites do not have the violet absorption. Clearly there is a lot here for Mr. Brown to explain in his study of these interesting little satellites, and it seems clear that his new data set will provide the necessary fundamental information for the investigation.

\section{Physical Properties}

Apart from the compositional conclusions derived from the information given above, there is no direct information on the physical properties of the Uranian satellites. We cannot yet measure their masses, their diameters, geometric albedos, temperatures, or figures. Some of this information can be pieced together from a string of inferences, as we shall see, but first I will review what is known about the masses of the satellites based 
on relatively recent theoretical discussions and on new observational programs in progress.

Greenberg (1975, 1976, 1979) has given the most recent thorough summary of the situation with regard to the satellite masses and has reviewed all the earlier literature. Greenberg analyzes the Laplace relation among the satellites and shows in a second-order perturbation theory that the products of the masses of certain satellite combinations can be found to establish certain constraints. The results are, for masses denoted $m_{1} \ldots m_{5}$ for the satellites Ariel through Miranda,

$$
\begin{aligned}
& \mathrm{m}_{1} \cdot \mathrm{m}_{2} \leqslant 10^{-9} \\
& \mathrm{~m}_{5} \cdot \mathrm{m}_{1} \lesssim 5 \times 10^{-12} \\
& \mathrm{~m}_{5} \cdot \mathrm{m}_{2} \lesssim 6 \times 10^{-12}
\end{aligned}
$$

in units of the mass of Uranus. Dr. Rattier at this conference has used new observations of Miranda's precession to derive

$$
\mathrm{m}_{1} \cdot \mathrm{m}_{2}=(1.10 \pm .25) \times 10^{-10}
$$

and finds $m_{3}=1.5 \times 10^{-4}$ from new data based on 200 plates of Miranda. Also at this conference, Dr. Ferraz-Mello has quoted the value $m_{3}=(1.47 \pm .10) \times 10^{-4}$ from the 1981 work of Veillet. It has been gratifying to learn at this conference that there is renewed interest in determining the masses of the satellites from telescopic observations over a long time base and that both the observational and theoretical work are now going on at a rapid pace.

We can approach the problem of the masses of the satellites from another viewpoint as we try to understand simultaneously the radii, mean densities, and surface geometric albedos of these bodies. The radius, $r$, of a Uranian satellite is related to its geometric albedo, $\mathrm{p}_{V}$, and observed stellar magnitude, $\mathrm{V}(1,0)$, by

$$
5 \log r=V_{\odot}-V(1,0)-2.5 \log \mathrm{P}_{\mathrm{V}}
$$

where $V_{\odot}$ is the stellar magnitude of the sun, -26.77 . The mass is $\mathrm{m}=\frac{4}{3} \pi \mathrm{r}^{3} \bar{\rho}$ where $\bar{\rho}$ is the mean density in $\mathrm{g} / \mathrm{cm}^{3}$.

We have established the presence of water ice or frost on the surface of four of the satellites and can presume that it also exists on Miranda. We have also given evidence that the spectral 
reflectances are rather neutral, both of which pieces of information suggest a relatively high geometric albedo comparable to snow (about 0.6 or greater). The close similarity to Ganymede tends to argue for a similar geometric albedo $(0.43)$ to that satellite. In all, the best estimate of $\mathrm{p}_{V}$ for the Uranian satellites is in the range 0.4-0.6. Further, studies of the icy satellites of Jupiter and Saturn (particularly the latter) show mean densities in the range $1-2 \mathrm{~g} / \mathrm{cm}^{3}$. A reasonable estimate for the icy satellites of Uranus might be $1.3 \mathrm{~g} / \mathrm{cm}^{3}$, the same as Saturn's Rhea.

In Table IV, I give a sample set of calculations of the radii of the Uranian satellites for different values of the geometric albedo. For the purposes of further discussion here we can assume $\mathrm{P}_{\mathrm{V}}=0.5$, giving radii in the range $160-520 \mathrm{~km}$.

TABLE IV. RADII (KM) OF THE URANIAN SATELLITES

\begin{tabular}{lrrrrrr}
\hline & \multicolumn{7}{c}{$\mathrm{p}_{\mathrm{V}}$} \\
\cline { 2 - 7 } & 0.1 & 0.2 & 0.4 & 0.5 & 0.6 & 0.8 \\
\hline U5 Miranda & 360 & 260 & 180 & 160 & 150 & 130 \\
U1 Arie1 & 1050 & 740 & 520 & 470 & 430 & 370 \\
U2 Umbrie1 & 710 & 500 & 360 & 320 & 290 & 250 \\
U3 Titania & 1150 & 810 & 580 & 520 & 470 & 410 \\
U4 Oberon & 1020 & 720 & 510 & 460 & 420 & 360 \\
\hline
\end{tabular}

In the verbal presentation of this paper at Bath, I suggested that the spectrophotometric evidence favored a slightly lower value of $\mathrm{p}_{V}$ for Umbriel, vis. 0.3. The new observations obtained since the Bath conference do not support that contention, and there is presently no evidence for differences among the four satellites so far observed in the near infrared. We thus use $p_{V}=0.5$ for all of the objects. If we then assume $\bar{\rho} 1.3 \mathrm{~g} / \mathrm{cm}^{3}$, we can calculate the masses corresponding to the diameters derived above and examine them for compliance with the Greenberg criteria and the new derivations referenced above. It will be seen in Table $\mathrm{V}$ that the Greenberg mass constraints can easily be met for all values of the radius derived from $0.4 \leqslant p_{\mathrm{V}} \leqslant 0.6$. 
TABLE V. MASSES OF THE URANIAN SATELLITES ${ }^{a}$

\begin{tabular}{lcll}
\hline & \multicolumn{3}{c}{$\mathrm{p}_{\mathrm{V}}$} \\
\cline { 2 - 4 } & 0.4 & 0.5 & 0.6 \\
\hline U5 Miranda & 0.37 & 0.26 & 0.21 \\
U1 Ariel & 8.8 & 6.5 & 5.0 \\
U2 Umbrie1 & 2.9 & 2.1 & 1.5 \\
U3 Titania & 12.3 & 8.8 & 6.5 \\
U4 Oberon & 8.3 & 6.1 & 4.7 \\
\hline assumes $\bar{p}=1.3 \mathrm{~g} / \mathrm{cm}^{3}$. & Al1 masses in units \\
10 &
\end{tabular}

Dr. Rattier's estimate of $m_{3}$ is not within the range found from the considerations discussed, requiring a rather large mean density and lower albedo, though his value of $m_{1} \cdot m_{2}$ can be met with only a slightly lower albedo and/or higher mean density than the preferred range noted. Clearly, additional work is needed, and the assumptions upon which this simple analysis is based need refinement, but we see that most of the constraints can be met for plausible ranges of the basic physical parameters. We also see that the basic discovery of water ice on these satellites is very important in establishing them as objects of rather high albedo, hence relatively small size. For $\mathrm{p}_{V}=0.5$, Table IV shows that the Uranian satellites are comparable in radius to the largest asteroids and the satellites of Saturn (except Titan).

\section{Origin of the Uranian Satellites}

The peculiar dynamical state of Uranus and its satellites has been the focus of most studies of the origin of this system to date. Uranus has an obliquity of about $98^{\circ}$ and the satellite system is very regular (mostly circular orbits of very low inclination) and lying in the planet's equatorlal plane. Any theory of the origin of the satellites must take into account the mechanism and time scale for the tilting of the planet itself, the presumption being that Uranus condensed with its spin axis nearly perpendicular to the ecliptic, followed by a tilting event. Singer 
(1975) has considered several scenarios and favors the formation or acquisition of the satellites after the planet tilted. He suggests the tidal capture of a body having $5-10 \%$ of the planet's mass, after which the body broke up and some $1 \%$ of it remains in the form of the five observed (and perhaps some unobserved) satellites.

From the point of view of composition some remarks can be made. The rings of Uranus appear to be composed of dark particles (Sinton 1977; Smith 1977), forming yet another contrast with the system of Saturn's satellites and rings. The total mass of the Uranian rings is probably on the order of $10^{-12}$ the mass of the planet, and thus far less than that of even Miranda. The satellites appear to be composed of volatile material, water ice. This difference in the composition of ring and satellite material may support Singer's hypothesis and may represent some degree of spatial differentiation of material from a disrupted body that provided the matter from which the satellites originated. Dr. Podolak has presented other information and ideas on the origin of Uranus and the satellites at this conference.

\section{The Future}

Continued improvements in observational equipment will permit further ground-based studies of the satellites and rings of Uranus, perhaps giving a clearer understanding of the interrelation between the two systems, both dynamically and chemically. Additional satellites may be found, particularly closer to the planet and perhaps intimately associated with specific rings in the ninecomponent (at least) system.

The Voyager 2 flyby of the Uranus system In 1986 will afford brief and imperfect glimpses of some of the satellites, particularly Miranda and Ariel, but the approach and viewing geometry are not optimum for a satellite-centered mission as a consequence of the planet's tilt. Still, the first close look at any components of the Uranian system is likely to help us understand the aggregate, and is anticipated with the greatest enthusiasm. 


\section{ACKNOWLEDGEMENT}

This research and travel to the Bath conference were supported in part by NASA Grant NGL 12001-057. I thank R. H. Brown for his contribution to this work.

\section{REFERENCES}

ALEXANDER, A. F. O'd. 1965. The Planet Uranus (New York: American Elsevier) $300 \mathrm{pp}$.

ANDERSSON, L. E. 1974. A photometric study of Pluto and satellites of the Jovian outer planets. Ph.D. thesis, Univ. of Indiana, Bloomington.

BARTON, S. G. 1946: The names of the sateliites. Pop. Astron. 54, 122-131.

BELL, J. F., CLARK, R. N., McCORD, T. B., and CRUIKSHANK, D. P. 1979. Reflection spectra of Pluto and three distant satellites. BulZ. Amer. Astron. Soc. 11, 572 (Abstr.).

CLARK, R. N. 1980. Ganymede, Europa, Callisto, and Saturn's rings: Compositional analysis from reflectance spectroscopy. Icarus $44,388-409$.

CLARK, R. N., and McCORD, T. B. 1980a. The Galilean satellites: New near-infrared spectral reflectance measurements $(0.65$ $2.5 \mu \mathrm{m})$ and a $0.325-5 \mu \mathrm{m}$ summary. Icarus $41,323-339$.

CLARK, R. N., and McCORD, T. B. 1980b. The rings of Saturn: New near-infrared reflectance measurements and a $0.326-4.08 \mu \mathrm{m}$ summary. Icarus 43, 161-168.

CRUIKSHANK, D. P. 1980. Near-infrared studies of the satellites of Saturn and Uranus. Icarus 41, 246-258.

CRUIKSHANK, D. P., and BROWN, R. H. 1981. The Uranian satellites: Water ice on Ariel and Umbriel. Icarus 45, 607-611.

CRUIKSHANK, D. P., PILCHER, C. B., and MORRISON, D. 1977. Identification of a new class of satellites in the outer solar system. Astrophys. J. 40, 1006-1010.

CRUIKSHANK, D. P., STOCKTON, A., DYCK, H. M., BECKLIN, E. E., and MACY, W. Jr. 1979. The diameter and reflectance of Triton. Icarus 40, 104-114.

DEGEWIJ, J., ANDERSSON, L. E., and ZELLNER, B. 1980a. Photometric properties of outer planetary satellites. Icarus 44, 520-540.

DEGEWIJ, J., CRUIKSHANK, D. P., and HARTMANN, W. K. 1980 b. Nearinfrared colorimetry of J6 Himalia and S9 Phoebe: A summary of 0.3- to 2.2- $\mu \mathrm{m}$ reflectances. Icarus 44, 541-547.

GONDOLATSCH, F. 1965. In Landolt-Bormstein Tables, New Series, eds. K. H. Hellwege and H. H. Voigt, vol. 1 (Berlin: Springer-Verlag) p. 150 .

GREENBERG, R. 1975. The dynamics of Uranus' satellites. Icarus 24. 325-332.

GREENBERG. R. 1976. The Laplace relation and the masses of Uranus' satellites. Icarus 29, 427-433.

GREENBERG, R. 1979. The motions of Uranus' satellites: Theory and application. In Dynomics of the Solar System, ed. R. L. Duncombe (Dordrecht: Reide1) pp. 177-180. 
HARRIS, D. L. 1961. Photometry and colorimetry of planets and satellites. In Planets and Satellites, eds. G. P. Kuiper and B. M. Middlehurst (Chicago: Univ. of Chicago Press) pp. 272-342.

HARTMANN, W. K., CRUIKSHANK, D. P., DEGEWIJ, J., and CAPPS, R. W. 1981. Surface materials on unusual planetary object Chiron. Icarus, submitted.

JOHNSON, P. E., GREENE, T. F., and SHORTHILL, R. W. 1978. Narrowband spectrophotometry of Ariel, Umbriel, Titania, Oberon, and Triton. Icarus $36,75-81$.

KUIPER, G. P. 1949. The fifth satellite of Uranus. Publ. Astron. Soc. Pacific 61, 129.

MORRISON, D., and CRUIKSHANK, D. P. 1974. Physical properties of the natural satellites. Space Sci. Rev. 15, 641-739.

MORRISON, D., CRUIKSHANK, D. P., PILCHER, C. B., and RIEKE, G. H. 1976. Surface compositions of the satellites of Saturn from infrared photometry. Astrophys. J. Lett. 207, L213-L216.

NICHOLSON, P. D., and JONES, T. J. 1980. Two-micron spectrophotometry of Uranus and its rings. Icams 42, 54-67.

REITSEMA, H. J., SMITH, B. A., and WEISTROP, D. E. 1978. Visua1 and near-infrared photometry of the Uranian satellites. Bull. Amer. Astron. Soc. 10, 585 (Abstr.).

SINGER, S. F. 1975. When and where were the satellites of Uranus formed? Icarus 25, 484-488.

SINTON, W. M. 1972, A near-infrared view of the Uranus system. Sky Telesc. 44, 304-305.

SINTON, W. M. 1977. Uranus: The rings are black. Science 198, 503-504.

SMITH, B. A. 1977. Uranus rings: An optical search. Nature 268,32 .

WHITAKER, E. A., and GREENBERG, R. J. 1973. Eccentricity and inclination of Miranda's orbit. Mon. Not. R. Astron. Soc. $165,15 \mathrm{P}-18 \mathrm{P}$.

\section{NOTE:}

After the Bath conference and following completion of the above text, some new results on the infrared spectra of Titania, Oberon, and Umbriel were published by Soifer et al. * They used the Hale 5-m telescope to obtain spectra in the $1.5-2.5 \mu \mathrm{m}$ region as presented here, and their results confirm the identification of water ice or frost on the surfaces of the three satellites studied. For reasons described in their paper, they favor very low geometric albedos for the Uranian satellites.

* SOIFER, B. T., NEUGEBAUER, G., AND MATTHEWS, K. 1981. Nearinfrared spectrophotometry of the satellites and rings of Uranus. Icams 45, 612-617. 